\title{
ANN based Battery Health Monitoring - A Comprehensive Review
}

\author{
Sai Vasudeva Bhagavatula ${ }^{l}$, Venkata Rupesh Bharadwaj Yellamraju², Karthik Chandra Eltem³, \\ Phaneendra Babu Bobba ${ }^{4}$, and Naveenkumar Marati ${ }^{5}$
}

\author{
${ }^{1}$ Dept. of Electrical \& Electronics Engineering, GRIET, Hyderabad, Telangana., India \\ ${ }^{2}$ Dept. of Electrical \& Electronics Engineering, GRIET, Hyderabad, Telangana., India \\ ${ }^{3}$ Dept. of Electrical \& Electronics Engineering, GRIET, Hyderabad, Telangana., India \\ ${ }^{4}$ Dept. of Electrical \& Electronics Engineering, GRIET, Hyderabad, Telangana., India \\ ${ }^{5}$ PES- Hardware, Valeo India Private Limited, Chennai, Tamilnadu, India
}

\begin{abstract}
The development of electric vehicles has bought a great revolution in the field of battery management as it deals with the health of the battery and also the protection of the battery. State of Charge (SoC) and State of Health $(\mathrm{SoH})$ are the important parameters in determining the battery's health. Advancements in Artificial Neural Networks and Machine Learning, a growing field in recent years has bought many changes in estimating these parameters. Access to huge battery data has become very advantageous to these methods. This manuscript presents an overview of different Artificial Neural Network techniques like Feedforward Neural Network (FNN), Extreme Learning Machine (ELM), and the Long Short Term Memory (LSTM). These techniques are trained with already existing data samples consisting of different values of voltages, currents at different temperatures with different charging cycles and epochs. The errors in each technique are different from the other as the constraints in one method are rectified using the other method to get the least error percentage and get the nearest estimate of the SoC and SOH. Each method needs to be trained for several epochs. This manuscript also presents a comparison of different methods with input parameters and error percentages.
\end{abstract}

\section{Introduction}

A Battery Management System (BMS) has brought a great revolution in the field of Electric Vehicles(EVs), smart cities, health care, and other electronic devices. A BMS consists of a protection circuit for a system against over current, over voltage, controlling charging and discharging of a battery pack, maintaining cell and pack temperature, and many other parameters. A Battery Monitoring System is a circuit that takes arguments or parameters such as voltage, current, and temperature used by the BMS, for estimating the $\mathrm{SoC}$ and $\mathrm{SoH}$ which gives us information about the battery's Health and its charging percentage. Artificial Neural Networks being a subset of machine learning need to be trained using different learning techniques for the accurate prediction and estimation of performance characteristics. Fig. 1 shows the block diagram of how one can estimate the $\mathrm{SoC}$ and $\mathrm{SoH}$.

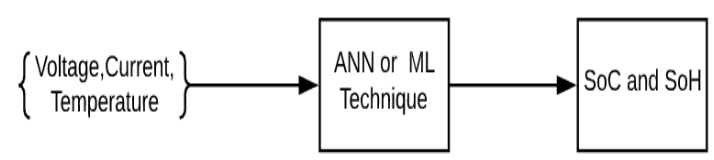

Fig 1. Block diagram of ANN for $\mathrm{SoC}$ and $\mathrm{SoH}$

The $\mathrm{SoC}$ and $\mathrm{SoH}$ of a battery are correlated with the battery's voltage, current, and temperature this paper presents a comparison of different ANN techniques used for the Estimation of SoC and SoH. Fig.2 is a basic summary of different ML techniques for the estimation of $\mathrm{SoC}$ and $\mathrm{SoH}$. 


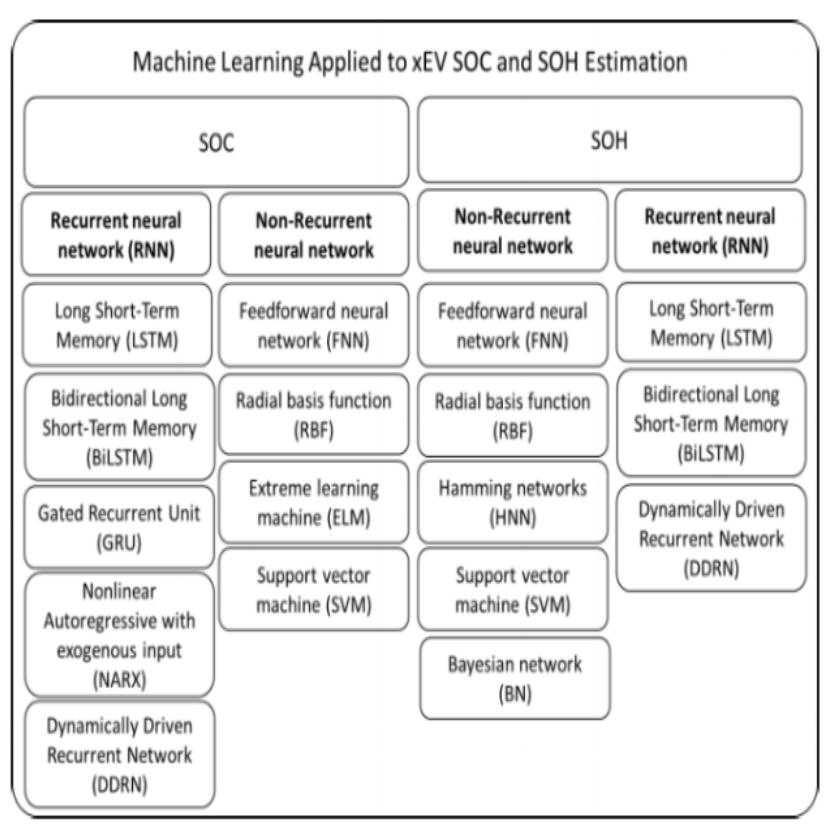

Fig 2. Different $\mathrm{ML}$ techniques for $\mathrm{SoC}$ and $\mathrm{SoH}$ estimation [1]

\section{Feedforward Neural Network}

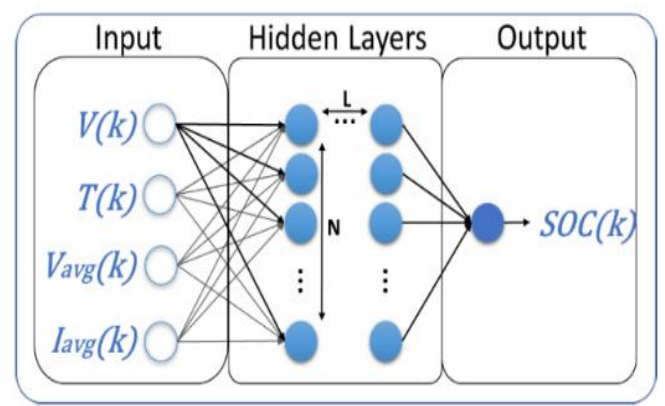

Fig 3. FNN Architecture for SoC [1]

FNN (Feed forward Neural Network) is the basic method of all the ANN techniques, is simple to understand the process is unidirectional, and moves only in the forward direction so it is called Feedforward. Estimation of SoC and $\mathrm{SoH}$ using the FNN technique given by the authors[2]. The performance characteristics such as SoC and $\mathrm{SoH}$ depend on the voltage, current, temperature[3], and also capacity of the cell. The battery terminal voltage had a significant effect on the estimation of SoC[4] given by the authors in [5]. The authors of [1] depict a feedforward-based model for the estimation of SoC according to the authors of the paper already trained datasets are taken for the estimation. The $\mathrm{SoC}$ value is given by

$\mathrm{D}=\left\{\left(\psi(1), \operatorname{SoC}(1)^{*}\right),\left(\psi(2), \operatorname{SoC}(2)^{*}\right), \ldots,(\psi(\lambda)\right.$,

$$
\left.\operatorname{SoC}(\lambda)^{*}\right\} \text {, }
$$

' $\lambda$ ' is the size of the pulse. The ideal representation of SoC $(y)^{*}$ and $\psi(y)$ being state-of-charge value and the vector representing the pulse input respectively.

Fig.4 and Fig 5 shows the results acquired for FNN based SoC Estimation a) SoC vs Percentage Error b) Epochs vs Mean Absolute Error. Where the estimation error is shown as a function of the true SOC values or ground-truth values.

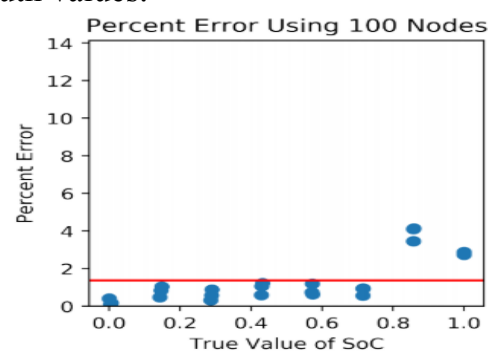

(a)
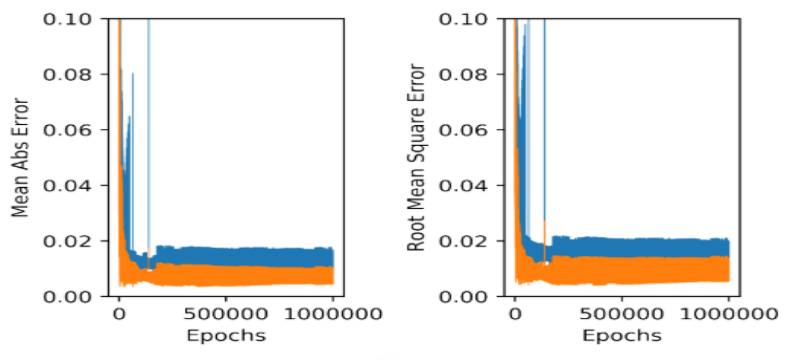

Fig 4. Estimation of SoC (FNN) for 100 Nodes[6]

Fig. 4. for 100 nodes the mean error is below in the range of $2 \%$ as shown in fig 4(a) and fig 4(b), the System is trained for 10,000 epochs.

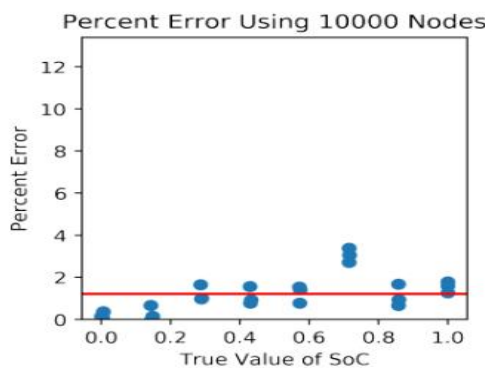

(a)
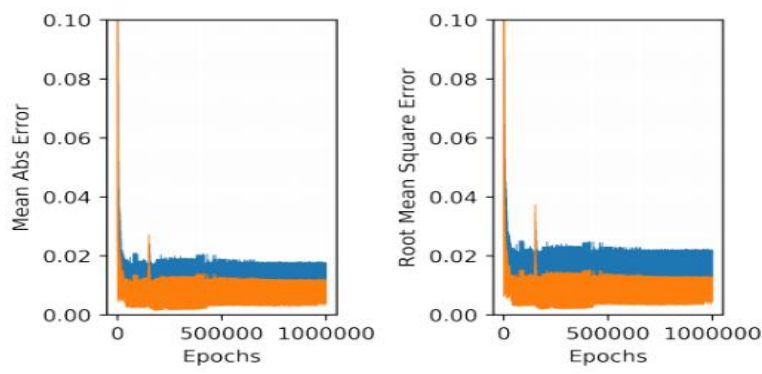

(b)

Fig 5. Estimation of SoC (FNN) for 1000 Nodes.[6]

Fig. 5 shows the results for 1000 nodes the mean error is also below in the range of $2 \%$ from Fig 5(a) and Fig.5(b),the system is trained using 10,000 epochs But the compared to the MAE of 100 nodes the 10000 nodes were more accurate so we can say that the number of nodes and the epochs affects the accuracy of the FNN. From [7] the authors applied similar method to FNN with input time-delays having a single hidden layer with 10 nodes. Later multiple time-delayed input signals were, applied and practical considerations such as the effect of capacity estimation error on SOC estimation was studied. 
SoH Estimation using FNN

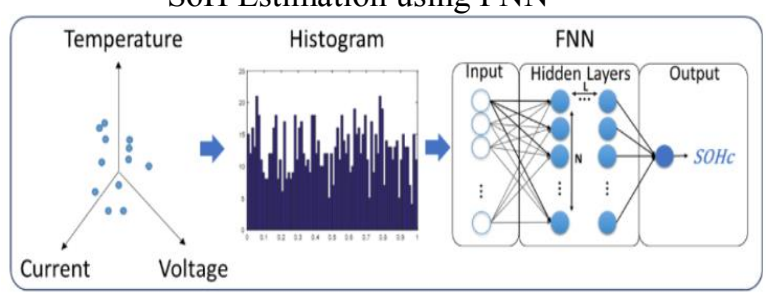

Fig 6. Network model for SoH Estimation [1]

Fig. 6 shows the network model for the estimation the input parameters for the $\mathrm{SoH}$ is the same as for $\mathrm{SoC}$ so there is a direct relation between $\mathrm{SoC}$ and $\mathrm{SoH}$. The charging and discharging profiles and the fully charged voltage values which give us the information about the Health Percentage. Similar to SoC FNN also determines the estimated SoH of a lithium ion cell[8]. a model has been introduced based on EIS and neural networks for the monitoring of $\mathrm{SoH}$ and can predict the degradation in the performance of the battery performance[9]. Using the first order equation of ECM an NN method is used in order to estimate the SoH[9][10]. The SoHr has a correlation with the terminal voltage the authors from [11]. The authors of [12] showed that the mean voltage deviation for $\mathrm{SOHr}$ at $25^{\circ} \mathrm{C}$ at 13,000 and $80,000 \mathrm{Km}$ respectively are $0.32 \%$ and $0.28 \%$.

\section{Extreme Learning Machine(ELM)}

ELM is a machine learning technique used to estimate battery health and SoC. It is closely related to the feedforward neural network where it is a single layer FNN. It has a considerable advantage over FNN because of its unique learning technique and it does not have time-consuming processes like Back Propagation(BP) algorithms as in FNN. Instead, it uses more Penrose pseudo inverse matrix [13]. Frequently used Health indicators in any $\mathrm{NN}$ or regression techniques are internal resistance, total capacity, and temperature. Generally, a filtering technique is used with ELM to obtain the final approximate. In [14] a gravitational search algorithm is employed with ELM to get the best estimates of SoC for the number of neurons in the hidden layer to obtain results required. It proves that ELM has a very low computational load and highly accurate with RMSE with less than $2 \%$ with any sort of driving profile compared to other techniques like Back Propagation and Radial Basis Neural Network(RNN). ELM is also employed in many SoH estimators as in [15] with a novel technique used, where the health indicators such as Ohmic internal resistance and Polarized internal resistance. ELM which is developed to nullify the disadvantages of FNN and its backpropagation techniques has a significant advantage in accuracy when compared to its similar one.
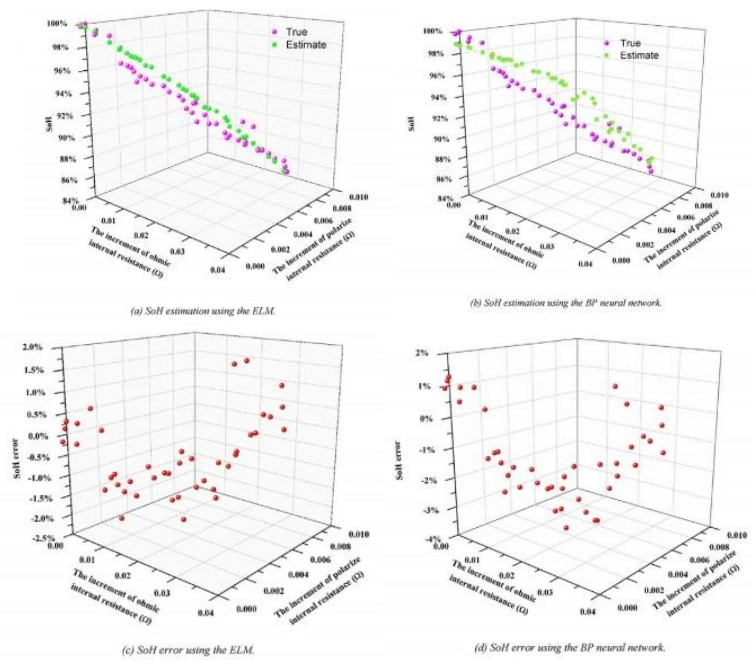

Fig 7. Estimation of $\mathrm{SOH}$ using neural networks [4]

The ELM and BP neural network both can achieve accurate results for estimating $\mathrm{SOH}$ as seen in (Fig 7a \& 7b) However the SoH estimated using the ELM neural network [15] can track battery degradation more accurately than BP neural can. The error margin for ELM is also less compared to BP neural network, hence the ELM has higher estimation accuracy than BP neural network. The error margin for both methods is calculated. The margin of SoH error in ELM (Fig 7c) is in range of $[-2.5 \%, 2 \%]$ and for BP (Fig 7d) error margin is in range of $[-4.0 \%, 2 \%]$, which indicates that ELM has more accuracy than BP neural network.

\section{Long Short Term Memory(LSTM)}

Long short term Memory Neural network is a special kind of Recurrent neural network. Generally, recurrent neural networks are very useful time series problems because of their capability of retaining past information. But there is a significant drawback in RNN such as error explosion and vanishing[16] during the backpropagation in a closed loop.

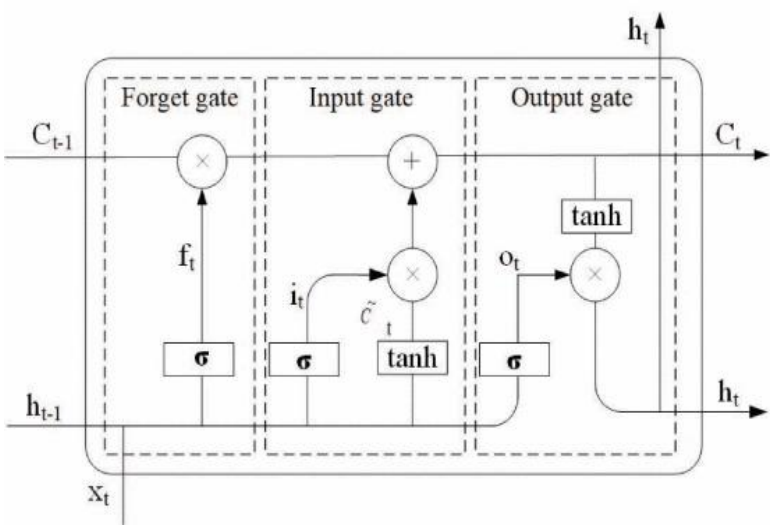

Fig 8. Architecture of the LSTM cell [6] 


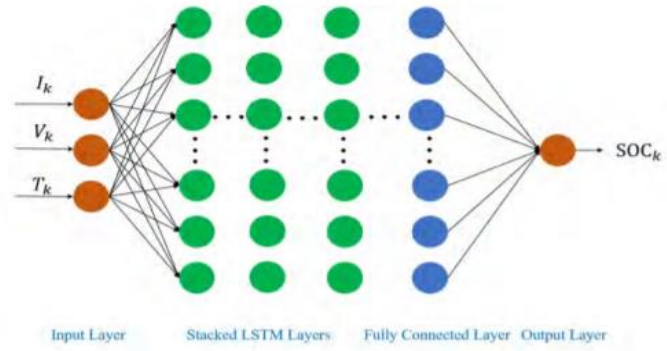

Fig 9. Neural Network Model of LSTM [16]

RNN has comparatively less time dependency i.e., can only retain the information of the immediate past but in contrast LSTM emerged as a winner because of high time dependency. Due to this notable advantage in time series problems such as $\mathrm{SoC}$ and $\mathrm{SoH}$ prediction in Battery Management System [17] and Speech recognitions LSTM NN have become more popular compared to its counterpart. $\psi_{\mathrm{k}}$ and $\mathrm{h}_{\mathrm{k}-1}$ are the 2 inputs fed to the LSTM where $\psi_{k}$ consists of $\left[V_{k} I_{k} T_{k}\right]$ as a part and the $h_{k}$ is the output of the hidden layer in the previous instant [18]. A stacked LSTM in comparison with UKF is proposed for SoC estimation [19] which makes use of 50 nodes and 3 hidden layer configuration (Fig.9) establishing an accurate relationship between time dependency and SOC. Nearly 7000 samples of data are taken from DST,US06, and FUDS tests for the training of the Neural network. Achieving an overall maximum absolute error of $2 \%$ with RMSE $1.07 \%$ and MAE nearly $0.84 \%$ with known initial SOC making it a viable method to use. But it's not possible in practical application but it converges to actual values in due course of time.
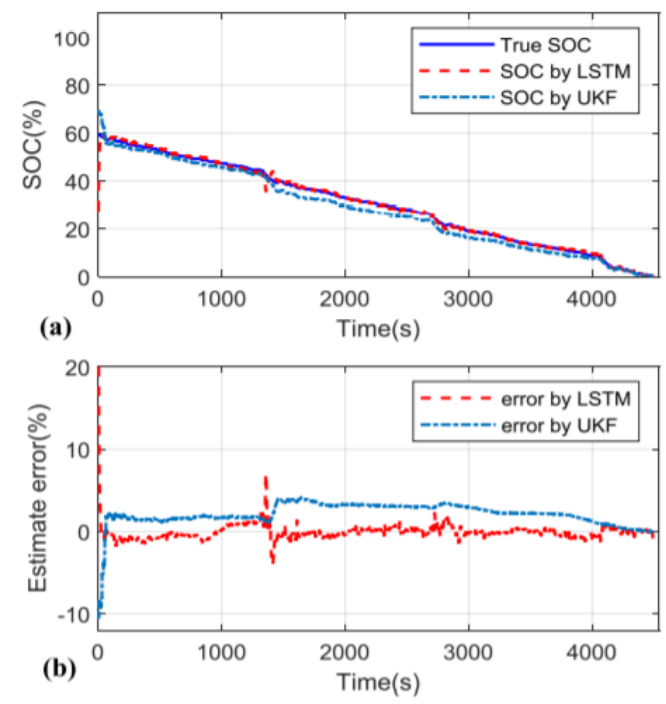

Fig 10. Comparison results of LSTM and UKF when initial $\mathrm{SoC}$ is $60 \%$ [19]

As far as results suggest the proposed method is highly accurate than Unscented Kalman Filter (UKF) except when the initial $\mathrm{SoC}$ is $100 \%$. In Fig. 10 and Fig. 11 it is clear that when moving to $60 \%$ and $80 \%$ initial SoC results one can see the error rate of UKF is high when compared to LSTM.
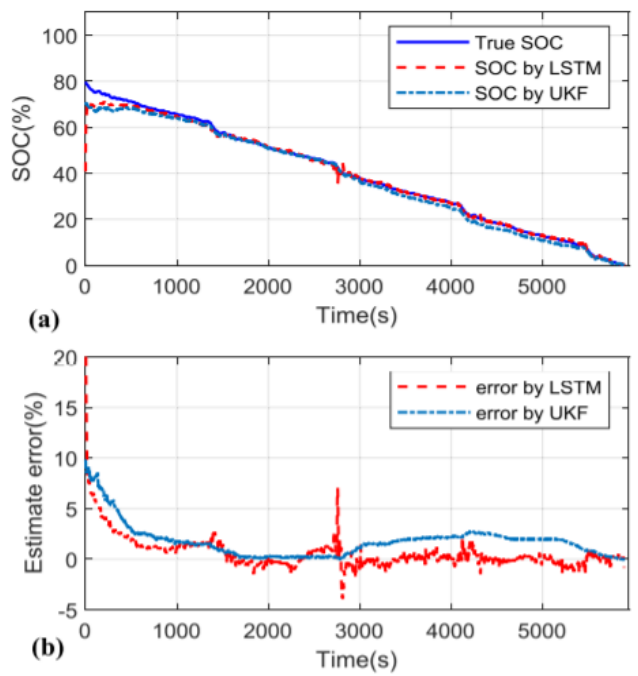

Fig 11. Comparison results of LSTM and UKF when initial $\mathrm{SoC}$ is $80 \%$. [19]

A significant advancement to the LSTM is bidirectional LSTM (BiLSTM). The major difference between the unidirectional and bidirectional LSTMs is that in bidirectional the output from $h_{k}$ is used to compute the $\mathrm{h}_{\mathrm{k}-1.1}$.The authors of the manuscript [20] proposed a similar method and they suggest that 64 hidden neuron configuration gives the best results and also shows that LSTM stands supreme to all other RNN neural networks based on their computational load results and error rates as in Fig 12 and Fig 13.
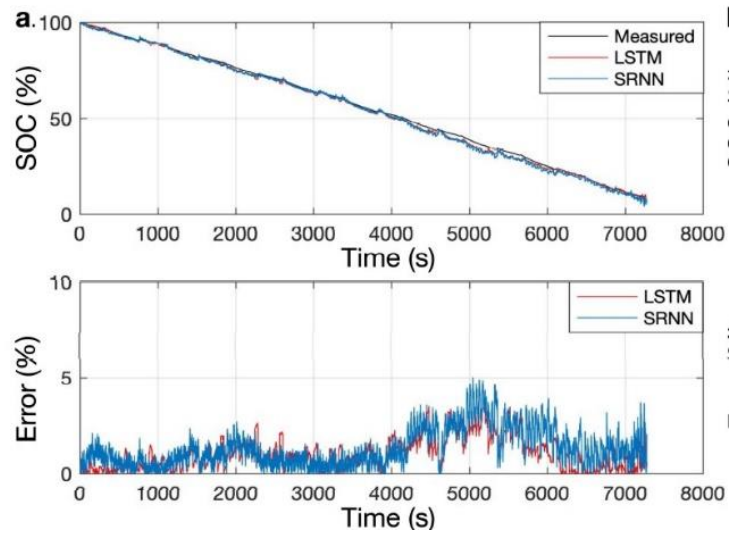

Fig 12. Error comparison of BiLSTM and SRNN [20]
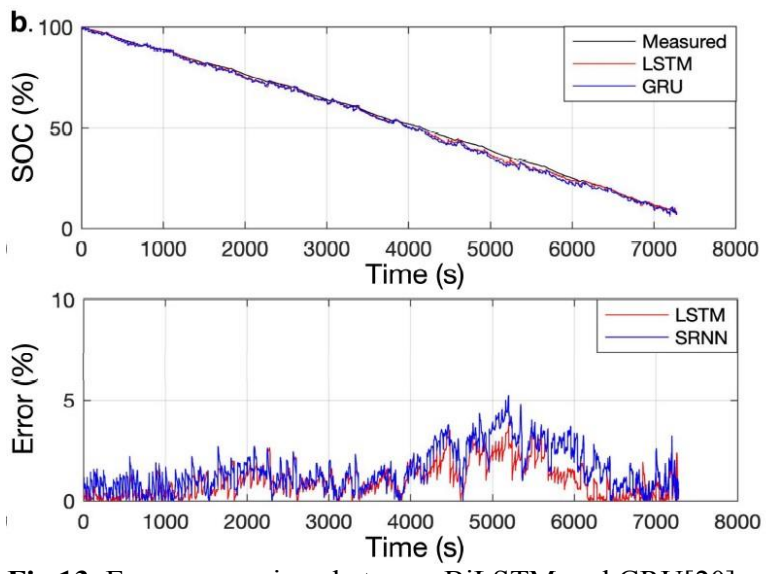

Fig 13. Error comparison between BiLSTM and GRU[20] 
Table 1 Comparison of Different ANN Techniques

\begin{tabular}{|c|c|c|c|c|c|}
\hline S.NO & Technique & Error Rate & Driving Profile & Temperature & $\begin{array}{l}\text { Reference } \\
\text { No }\end{array}$ \\
\hline 1 & $\begin{array}{l}\text { Long Short term } \\
\text { Memory(LSTM) } \\
\text { /UKF }\end{array}$ & $\begin{array}{l}\text { 100\% Initial SoC } \\
\text { 1.07\%/0.42\%@RMSE } \\
\text { 0.84\%/0.36\%@MAE } \\
\text { 60\% Initial SoC } \\
\text { 1.07\%/2.54\%@RMSE } \\
\text { 0.53\%/2.28\%@MAE }\end{array}$ & DST,US06 Dual Training Data & - & [19] \\
\hline 2 & Bi LSTM & $\begin{array}{l}\text { US06/HWFET@MAE } \\
0.56 \% / 0.46 \% @ 25^{\circ} \mathrm{C} \\
0.98 \% / 0.80 \% @ 10^{\circ} \mathrm{C} \\
0.95 \% / 0.54 \% @ 0^{\circ} \mathrm{C}\end{array}$ & US06,HWFET & $25^{\circ} \mathrm{C}, 10^{\circ} \mathrm{C} .0^{\circ} \mathrm{C}$ & [20] \\
\hline 3 & $\begin{array}{l}\text { Gated Recurrent } \\
\text { Unit }\end{array}$ & $\begin{array}{l}\text { US06/HWFET@MAE } \\
0.76 \% / 0.61 \% @ 25^{\circ} \mathrm{C} \\
1.05 \% / 0.84 \% @ 10^{\circ} \mathrm{C} \\
1.03 \% / 0.75 \% @ 0^{\circ} \mathrm{C}\end{array}$ & US06,HWFET & $25^{\circ} \mathrm{C}, 10^{\circ} \mathrm{C}, 0^{\circ} \mathrm{C}$ & [20] \\
\hline 4 & SRNN & $\begin{array}{l}\text { US06/HWFET@MAE } \\
0.93 \% / 0.75 \% @ 25^{\circ} \mathrm{C} \\
1.24 \% / 1.02 \% @ 10^{\circ} \mathrm{C} \\
1.15 \% / 0.78 \% @ 0^{\circ} \mathrm{C}\end{array}$ & US06,HWET & $25^{\circ} \mathrm{C}, 10^{\circ} \mathrm{C}, 0^{\circ} \mathrm{C}$ & [20] \\
\hline 5 & ELM-GSA & $\begin{array}{l}\text { BJDST/US06@25ㄷ } \\
0.76 \% / 1.56 \% @ R M S E \\
0.55 \% / 1.67 \% @ M A E \\
\text { BJDST/US06@45ㄷ } \\
0.68 \% / 1.2 \% @ \text { RMSE } \\
\text { 0.48\%/0.91\%@MAE }\end{array}$ & BJDST,US06 & $25^{\circ} \mathrm{C}, 45^{\circ} \mathrm{C}$ & [14] \\
\hline 6 & $\begin{array}{l}\text { (Extreme } \\
\text { Learning } \\
\text { Machine )ELM }\end{array}$ & $\begin{array}{l}\text { 1.72\%@MAE } \\
\text { 2.22\%@Maximum Error }\end{array}$ & NEDC & - & [15] \\
\hline 7 & $\begin{array}{l}\text { Feed Forward } \\
\text { Neural Network }\end{array}$ & $\begin{array}{l}\text { 0.33\%@RMSE@WLTC } \\
\text { 0.27\%@MAE@WLTC }\end{array}$ & US06,WLTC,NEDC,FTP75,GUDC & - & [2] \\
\hline 8 & FNN-BSA & $\begin{array}{l}\text { 0.81\%@RMSE@DST } \\
\text { 0.91\%@RMSE@FUDS }\end{array}$ & FUDS,DST & $0^{\circ} \mathrm{C}, 25^{\circ} \mathrm{C}, 45^{\circ} \mathrm{C}$ & [23] \\
\hline 9 & RNN & $\begin{array}{l}\text { SoHc:0.46\%@MSE } \\
\text { SoHr:0.29\%@MSE }\end{array}$ & - & $25^{\circ} \mathrm{C}, 45^{\circ} \mathrm{C}$ & [24] \\
\hline 10 & FNN & $\begin{array}{l}0.067 \text { Ah @RMSE } \\
0.17 \text { Ah@MAE }\end{array}$ & - & $25^{\circ} \mathrm{C}-60^{\circ} \mathrm{C}$ & [25] \\
\hline
\end{tabular}


When coming to the SoH estimations LSTM plays a crucial role where generally internal resistance [21]. Total capacity and sometimes SoC are employed in the models somehow differently a new estimation model is validated in [22] which uses I-V curves for the estimation based on the density of points on the I-V. It also brought a demerit with in the form of noise, even the slightest of noises are considered as aging in this model. The below is the comparison table for different ANN methods with their Error percentage for different driving profiles almost all of the below mentioned below got a percentage error below 2 percent. For the US06 driving profile, the long short term memory acquired the least error percentage using the Mean Absolute Error (MAE) of $0.36 \%$ at $100 \% \mathrm{SoC}$, whereas the same

\section{Conclusion:}

This manuscript presents a comparison of different ANN techniques used for the estimation of $\mathrm{SoC}, \mathrm{SOH}$, and the determination of the battery's health. The increasing amount of data determines the quality and efficiency of our required output. All the NN methods are trained with basic parameters like input voltages, currents, and temperatures but the error percentage in neural networks especially in the FNN method the number of nodes is also very important but the FNN method has a constraint on the error percentage over the ELM method since there is no feedback in the FNN method in giving higher error percentage in some cases whereas the ELM method is compared more efficiently since it is based on the pseudo inverse method so the time-consuming process is neglected in this method. In LSTM method due to the notable advantage time series problems such as $\mathrm{SoC}$ and $\mathrm{SoH}$ prediction in battery management system it is compared better than FNN and RNN The FNN method gave a mean absolute error of $0.27 \%$ at $25^{\circ} \mathrm{C}$, for the same temperature the ELM method gave an error percentage of $1.72 \%$. In the FNN method, the MAE depends on the number of epochs, for a 10,000 epochs FNN got $2 \%$ over MAE. So based on the results of different profiles and different temperatures the error of a particular $\mathrm{NN}$ method changes so it's uncertain to say which method gives the least error percentage.

\section{Future Scope}

More Advanced method like Fuzzy Neural Network is the most advanced method with the highest accuracy in estimating the performance characteristics. This method is the blend of both fuzzy logics and neural networks and doesn't require any mathematical model. It is very easy and involves easy interpretation and implementation unlike the conventional neural network methods the learning process is optional since it uses its own fuzzy rules

\section{References}

1. CARLOS.V, P.MALYSZ, K.PHILLIP, AND ALI EMADI, "Machine Learning Applied to method acquired an error percentage of 2.28 percent. All the methods gave the least error when the battery temperature is about $25^{\circ} \mathrm{C}$ which makes almost all the methods have a similar profile at absolute room temperature. The Extreme Learning Machine (ELM) method acquired for the NEDC driving profile got a 1.72 on the MAE with the Gravity Search Algorithm (GSA) the mean absolute error percentage has gone to 1.06 percent the feedforward neural network method gave a very appreciable result for the WLTC driving profile with the least error percentage of 0.27 percent for the MAE method and for the same driving profile it gave an error percentage of 0.33 for the Root Mean Square (RMS) error.

Electrified Vehicle Battery State of Charge and State Health and Estimation: State-of-the-Art", vol 8, pp.52796-52814,16 (Mar 2019)

2. C. Vidal, M. Haußmann, D. Barroso, P. M. Shamsabadi, A. Biswas,E. Chemali, R. Ahmed, and A. Emadi, "Hybrid energy storage system state-of-charge estimation using artificial neural networks for micro-hybrid applications" in Proc. IEEE ( August 2018).

3. C. Vidal, O. Gross, R. Gu, P. Kollmeyer, and A. Emadi, " $x E V$ Li-ion battery low-temperature effects-Review, ' IEEE Trans. Veh. Technol., vol. 68, no. 5, pp. 4560-4572, (May 2019.)

4. J. Yang, C. Du, T. Wang, Y. Gao, X. Cheng, P. Zuo, Y. Ma, J. Wang, G. Yin, J. Xie, and B. Lei, "Rapid prediction of the open-circuit-voltage of lithium ion batteries based on an effective voltage relaxation model, Energies", vol. 11, no. 12, p. 3444, (2018)

5. C. Chen, R. Xiong, R. Yang, W. Shen, and F. Sun, "State-of-charge estimation of lithium-ion battery using an improved neural network model and extended Kalman filter," J. Cleaner Prod., vol. 234, no. 5, pp. 1153-1164, (Oct. 2019).

6. W. Wang, Nicholas W. Brady, Chenyao Liao, Youssef A. Fahmy, E.Chemali, Alan C. West, and P.Matthias, "High-Fidelity State-of-Charge Estimation of Li-Ion Batteries using Machine Learning”, vol 1, (Aug 2019).

7. A. A. Hussein, "Capacity fade estimation in electric vehicle li-ion batteries using artificial neural networks," IEEE Trans. Ind. Appl., vol. 51, no. 3,pp. 2321-2330,(May 2015).

8. Hu, Xiaosong \& Li, Shengbo \& Yang, Yalian. "Advanced Machine Learning Approach for Lithium-Ion Battery State Estimation in Electric Vehicles". IEEE Transactions on Transportation Electrification, vol 2, (2015).

9. Duo Yanga, Yujie Wanga, Rui Pana, Ruiyang Chenb, Zonghai Chena. "A neural network based state-of-health estimation of lithium-ion battery in electric vehicles" ,The 8th International Conference on Applied Energy - ICAE, vol-105 (2016).

10. H. Lin, T. Liang and S. Chen, "Estimation of Battery State of Health Using a Probabilistic Neural Network," in IEEE Transactions on 
Industrial Informatics, vol. 9, no. 2, pp. 679-685, (May 2013).

11. J. Wu, Y. Wang, X. Zhang, and Z. Chen, "A novel state of health estimation method of li-ion battery using group method of data handling," J. Power Sour., vol. 327, pp. 457-464,( Sep. 2016).

12. D. Andre, A. Nuhic, T. Soczka-Guth, and D. U. Sauer, ' Comparative study of a structured neural network and an extended Kalman filter for state of health determination of lithium-ion batteries in hybrid electric vehicles,"' vol-26,(March 2013).

13. Suresh Kumar T, Sankar V, "Reliability improvement of composite electric power system using Unified Power Flow Controller", 2011 IEEE India Conference, INDICON, (2011).

14. M. S. Hossain Lipu, M. A. Hannan, A. Hussain, M. H. Saad, A. Ayob and M. N. Uddin, "Extreme Learning Machine Model for State-of-Charge Estimation of Lithium-Ion Battery Using Gravitational Search Algorithm," vol. 55, no. 4, pp. 4225-4234, (July-Aug. 2019).

15. Haihong Pan, Z. Lü, H. Wang, Haiyan Wei, Lin Chen, "Novel battery state-of-health online estimation method using multiple health indicators and an extreme learning machine" ,Volume 160, (2018).

16. R. Pascanu, T. Mikolov, and Y. Bengio, "On the difficulty of training Recurrent Neural Networks", in Proc. Int. Conf. Mach. Learn, Atlanta GA, USA, vol-28, pp. 1310-1318, (2013).

17. J.Qu, F.Liu, Y.Ma and J.Fan, "A Neural-NetworkBased Method for RUL Prediction and $\mathrm{SOH}$ Monitoring of Lithium-Ion Battery," in IEEE Access, vol. 7, pp. 87178-87191, (2019).

18. F. Yang, X. Song, F. Xu, and K.-L. Tsui, 'Stateof-charge estimation of lithium-ion batteries via long short-term memory network', IEEE Access, vol. 7, pp. 53792-53799, (2019).

19. E. Chemali, P. J. Kollmeyer, M. Preindl, and A. Emadi, "State-of-charge estimation of li-ion batteries using deep neural networks: A machine learning approach', vol. 400, pp. 242-255,(Oct. 2018).

20. C. Bian, H. He, and S. Yang, "Stacked bidirectional long short-term memory networks for state-of-charge estimation of lithium-ion batteries, Energy",vol. 191, Art. no. 116538. (Jan. 2020).

21. X. Tan et al., "Real-Time State-of-Health Estimation of Lithium-Ion Batteries Based on the Equivalent Internal Resistance," in IEEE Access, vol. 8, pp. 56811-56822, (2020), doi: 10.1109/ACCESS.2020.2979570.

22. Srinivas Rao J., Srinivasa Varma, P., Suresh Kumar. T, "Novel switching design structure for three phase 21-level multilevel inverter fed BLDC drive application", International Journal of Power Electronics and Drive Systems, vol. 9, no. 3, pp: 1202-1213, (2018)

23. M. A. Hannan, M. S. H. Lipu, A. Hussain, M. H. Saad, and A. Ayob, "Neural network approach for estimating state of charge of lithium-ion battery using backtracking search algorithm," IEEE Access, vol. 6, pp. 10069-10079, (2018).

24. A. Eddahech, O. Briat, N. Bertrand, J.-Y. Delétage, and J.-M. Vinassa, "Behavior and stateof-health monitoring of li-ion batteries using impedance spectroscopy and recurrent neural networks, ' ' Int. J. Electr.Power Energy Syst., vol. 42, no. 1, pp. 487-494, (Nov. 2012).

25. G.-W. You, S. Park, and D. Oh, 'Real-time stateof-health estimation for electric vehicle batteries: A data-driven approach, ' Appl. Energy, vol. 176, pp. 92-103, (Aug. 2016) 